\title{
Projections régionalisées de la hausse du niveau marin dans les territoires français d'outremer
}

\author{
Albéric BOTELLA ${ }^{1}$ \\ ${ }^{I}$ Département des Sciences de la Terre / Institut de l'Environnement, Université d'Ottawa, Canada-e-mail: abote036@uottawa.ca
}

\begin{abstract}
RÉSUMÉ. - Au cours des prochaines décennies, l'élévation du niveau des mers devrait contribuer à aggraver les effets des épisodes de submersion affectant le littoral français. Dans cette perspective, l'hypothèse d'une hausse globale du niveau marin de $60 \mathrm{~cm}$ à l'horizon 2100 a été adoptée dans la réglementation nationale relative au risque de submersion marine en 2011. En s'appuyant sur les projections établies par le GIEC en 2013, la présente étude montre que cette hypothèse est aujourd'hui sous-estimée.

Par ailleurs, les variations du niveau marin ne sont pas uniformes sur l'ensemble du globe. Notamment, les départements et collectivités d'outremer (DOM-COM), dispersés à travers les océans, seront défavorablement affectés par cette variabilité régionale. Dans le "scénario du pire" intéressant plus particulièrement les pouvoirs publics, la hausse du niveau marin d'ici 2100 demeurerait limitée à $100 \mathrm{~cm}$ sur la façade Atlantique de la métropole, mais serait proche de $120 \mathrm{~cm}$ à Mayotte ou La Réunion.

Sur la base de ce double constat, il est recommandé de mettre à jour l'hypothèse de travail retenue dans la réglementation et d'en augmenter la valeur de manière à refléter les plus récents travaux du GIEC. Considérant que les territoires ultramarins sont à la fois plus sensibles au risque de submersion marine et plus exposés à la hausse du niveau marin que la métropole, il est aussi proposé d'adopter des hypothèses de travail régionalisées.
\end{abstract}

Mots clefs : hausse du niveau marin, changement climatique, submersion marine, variabilité spatiale, GIEC

\section{Regional Projections of Sea-level Rise in French Overseas Territories}

\begin{abstract}
Over the coming decades, sea-level rise will increase the severity of flooding events impacting French coastal areas. Given this prospect, a sea-level rise of $60 \mathrm{~cm}$ by 2100 has been assumed and adopted in French regulations related to coastal flooding. The accuracy of this working hypothesis is considered here by comparing the adopted value with projections recently produced by the IPCC. Based on this comparison, it can be concluded that the risk is currently underestimated.

In addition, local sea-level changes can differ significantly from the global mean because of various spatial-dependant processes: gravitational and rotational effects influencing the redistribution of melting water from glaciers and ice sheets, dynamical steric effects affecting densities locally, and isostatic effects causing vertical land movements along coastlines. French overseas territories, which have a broad global distribution, will be adversly affected by this spatial variability. In the worst-case future climate change scenario - the one of most interest to decision-makers - sea-level would rise by less than $100 \mathrm{~cm}$ along the French Atlantic coast, compared to nearly $120 \mathrm{~cm}$ in Mayotte and La Réunion.

Given the two issues raised above, it is recommended that the national regulations are revised in order to reflect the most recent projections provided by the IPCC. Considering that French overseas territories are more vulnerable to flooding and could experience a more significant sea-level rise than French metropolitan coasts, it is proposed also to consider regional working hypothesis.
\end{abstract}

Key words: sea-level rise, climate change, coastal flooding, spatial variability, IPCC

\section{INTRODUCTION}

\section{I.1. Les spécificités du risque de submersion marine en outremer}

En 2010, la tempête Xynthia a durement frappé les côtes de la France métropolitaine, rappelant la nécessité d'une gestion durable de l'implantation humaine en zone littorale. Résultant de la conjonction d'une marée de vives-eaux et d'une forte tempête, cet épisode de submersion marine pourrait apparaître exceptionnel [Feuillet et al., 2012] ; la hausse progressive du niveau marin contribuera pourtant à augmenter la période de retour de tels événements. Cette analyse est généralisable à l'ensemble du territoire français, y compris aux Départements et Collectivités d'Outre-Mer (DOM-COM) qui se distinguent de la métropole par de nombreuses spécificités géographiques, socio-économiques et environnementales [Duvat et al., 2012]. En particulier, la vulnérabilité au risque de submersion apparaît élevée dans les territoires insulaires [Bellard et al., 2013], alors que les communautés humaines et les écosystèmes de la métropole disposent d'options plus variées pour s'adapter à la montée des eaux - y compris la possibilité de se replier dans les terres - et de ressources plus importantes pour développer leur résilience. Au regard de ces différences, il conviendrait donc d'estimer l'intensité de l'aléa auquel seront exposés les DOM-COM dans les décennies à venir. 
Pour répondre à ce besoin, l'expertise nationale s'appuie notamment sur les conclusions du groupe d'experts intergouvernemental sur l'évolution du climat (GIEC), qui, dans ses rapports successifs, a cherché à évaluer la hausse du niveau des mers à l'horizon 2100 en fonction de plusieurs scénarii. De manière récurrente, les projections globales établies par le GIEC sont reprises dans des travaux de recherches ou dans le cadre de l'information au public. Mais ces valeurs masquent une réalité physique plus complexe : les variations du niveau marin ne sont pas uniformes géographiquement, et le territoire maritime français, épars à travers le globe, sera nécessairement affecté par cette "variabilité régionale".

Le présent article vise à caractériser l'amplitude de cette variabilité en exploitant les plus récentes données compilées par le GIEC [IPCC, 2013 ; Church et al., 2013]. La prise en compte au plan national de ces projections sera évoquée en préambule, puis la méthodologie retenue par le GIEC pour évaluer les différentes contributions aux variations du niveau marin sera succinctement décrite. Enfin, des projections à l'horizon 2100 pour plusieurs sites d'outremer seront présentées et comparées aux hypothèses retenues à l'heure actuelle par les pouvoirs publics.

\section{I.2. Une évaluation en évolution permanente}

En 2010, dans le cadre de l'élaboration du Plan National d'Adaptation au Changement Climatique, l'Observatoire National sur les Effets du Réchauffement Climatique (ONERC) a recommandé la prise en compte d'une hausse du niveau marin de $60 \mathrm{~cm}$ à l'horizon 2100 [ONERC, 2010]. Cette hypothèse, dite "pessimiste", est basée sur les conclusions du quatrième rapport du GIEC [Meehl et al., 2007] ; elle a été définie comme la valeur haute des projections dans le scénario d'émissions de gaz à effet de serre (GES) le plus défavorable. La variabilité régionale apparaissant à l'époque difficile à évaluer et peu significative, une valeur unique a été proposée pour l'ensemble des côtes françaises, DOM-COM compris. Puis en 2011, le Ministère de l'Écologie et du Développement Durable a publié une circulaire précisant les modalités de prise en compte du risque de submersion marine en zone littorale [MEDDTL, 2011]. Servant de guide pour l'élaboration de mesures d'adaptation locales telles que la construction ou le renforcement d'ouvrages de protection, la délimitation de zones inconstructibles ou encore la définition de prescriptions pour les nouvelles habitations, ce document reprend in extenso les recommandations émises par l'ONERC. Une hausse de $60 \mathrm{~cm}$ pour l'ensemble de l'espace littoral français, nommée "aléa 2100", a alors été adoptée comme hypothèse de référence nationale.

Par ailleurs, la réglementation prévoit que cette valeur puisse être révisée en fonction de l'évolution des connaissances scientifiques sur les effets du changement climatique. Or depuis 2007, la compréhension des phénomènes physiques influant sur le niveau marin a évolué, tandis que les observations connaissent une amélioration à la fois qualitative et quantitative [Paragraphe E.6 - IPCC, 2013]. Ainsi la communauté scientifique est en mesure de mieux évaluer les incertitudes et de proposer des projections plus réalistes. Fort de ces avancées, le GIEC a publié un cinquième rapport d'expertise en septembre 2013 et a présenté de nouvelles fourchettes de projections, plus pessimistes que celles du précédent rapport (Figure 1). En effet, en considérant le scénario d'émissions de GES le plus défavorable, la hausse du niveau moyen global pourrait atteindre $98 \mathrm{~cm}$ d'ici la fin du siècle, estimation qui par ailleurs excède la valeur retenue pour l'aléa 2100.

\section{MODELISATION DE LA VARIABILITÉ RÉGIONALE}

Avant de détailler comment les différents processus concourant à la hausse du niveau marin ont été modélisés, il convient tout d'abord de rappeler deux notions physiques. Le niveau marin absolu, ou niveau géocentrique, représente la distance séparant la surface des océans et le centre de la Terre, et peut être mesuré directement par un satellite altimétrique ; le niveau moyen global est défini comme la moyenne du niveau marin absolu sur l'ensemble du globe. Le niveau marin relatif décrit quant à lui l'élévation de la surface des océans par rapport à un référentiel terrestre, par exemple une station marégraphique. Tandis que la hausse du niveau moyen global, mesure intégrée dans le temps et l'espace, est communément utilisée pour quantifier le réchauffement climatique, le niveau relatif constitue la grandeur pertinente pour évaluer localement le risque de submersion marine.

Trois causes majeures de variations du niveau marin relatif sont prises en compte dans les travaux du GIEC : les échanges de masse entre les océans et les continents, tels que la formation ou la fonte de glaces terrestres, ou encore le stockage d'eau sur les continents (contribution massique) ; les variations de volume des océans, principalement sous l'effet de la contraction ou l'expansion thermique et des variations locales de densité (contribution stérique) ; les mouvements verticaux de la croûte terrestre causés par les ajustements isostatiques (contribution isostatique). Les mouvements verticaux locaux causés par d'autres phénomènes tels que l'activité tectonique, la compaction des couches sédimentaires, le pompage excessif des nappes d'eau souterraine ou encore la charge volcanique n'ont pas été examinés par le GIEC et ne sont pas considérés dans cette étude.

Figure 1: Projections du niveau marin. Comparaison des projections d'élévation du niveau moyen global apparaissant dans le quatrième [Table 10.7 - Meehl et al., 2007] et le cinquième rapport [Table 13.5 - Church et al., 2013] du GIEC.

\begin{tabular}{|l|c|c|}
\hline Année de publication du rapport & AR4, 2007 & AR5, 2013 \\
\hline $\begin{array}{l}\text { Scénario d'émissions } \\
\text { le plus défavorable }\end{array}$ & SRES A1FI & RCP 8.5 \\
\hline $\begin{array}{l}\text { Période considérée } \\
\text { et durée moyenne de la période }\end{array}$ & $\begin{array}{c}\text { de 1980-1999 à 2090-2099 } \\
(105 \text { ans })\end{array}$ & $\begin{array}{c}\text { de 1986-2005 à 2100 } \\
(105 \text { ans) }\end{array}$ \\
\hline $\begin{array}{l}\text { Hausse moyenne globale (inter- } \\
\text { valle de confiance 5-95\%) }\end{array}$ & entre 26 et 59 cm & entre 52 et $98 \mathrm{~cm}$ \\
\hline
\end{tabular}




\section{II.1. Contribution massique}

Les glaces terrestres - glaciers et calottes glaciaires du Groenland et de l'Antarctique - constituent un volume d'eau considérable ; si elles venaient à fondre dans leur intégralité, le niveau moyen des océans s'élèverait de plus de 65 mètres. Bien que les glaciers représentent moins d'un pourcent de la masse totale de ces glaces, leur fonte a pourtant occasionné près d'un tiers de la hausse observée depuis 1971 [Table 13.1 - Church et al., 2013]. Localisés dans des régions plus chaudes que les calottes glaciaires, les glaciers répondent en effet plus rapidement aux changements climatiques. Néanmoins, la fonte des grandes calottes glaciaires devrait aussi s'accélérer au cours des décennies à venir, notamment par augmentation de la fonte superficielle ou évolution de la dynamique des glaces. Enfin, le changement climatique et les activités humaines génèrent des échanges entre les eaux continentales et les océans, qui affectent à leur tour le niveau marin. Quelle que soit leur nature, ces échanges de masse ne font pas varier le niveau marin de manière homogène, leur redistribution spatiale étant régie par plusieurs phénomènes physiques.

Tout d'abord, la surface des océans, en partie modelée par le champ gravitationnel local, est irrégulière. En particulier, les masses océaniques situées à proximité des glaces terrestres sont attirées par ces dernières ; la formation ou la disparition de glace entraîne donc une redistribution de ces masses liquides à la surface du globe, occasionnant des variations non uniformes du niveau marin [Tamisiea et al., 2003]. Prépondérants à proximité des calottes glaciaires et des grands glaciers, ces effets gravitationnels sont perceptibles jusqu'à plusieurs milliers de kilomètres de distance. A titre d'exemple, les projections établies par le GIEC pour le scénario RCP8.5 montrent que d'ici la fin du siècle, la calotte du Groenland pourrait perdre une quantité de glace équivalente à une hausse du niveau moyen global de 15 $\mathrm{cm}$; en l'absence d'autres contributions, cette fonte se traduirait localement par une baisse de $32 \mathrm{~cm}$ sur la côte nord de l'Islande, tandis qu'une hausse de $18 \mathrm{~cm}$ serait observée à Tahiti. De plus, ces redistributions de masse influent sur l'axe et la vitesse de rotation terrestre, ce qui affecte en retour la répartition des masses océaniques et donc la forme du géoïde [Milne et Mitrovica, 1998]. Non négligeables, ces effets rotationnels doivent aussi être pris en compte pour établir des projections régionales.

Pour déterminer la redistribution spatiale des masses océaniques occasionnée par la fonte des glaces terrestres, la méthode consiste dans un premier temps à estimer les variations de masse attendues pour chaque calotte glaciaire et chaque groupe de glaciers, de manière individuelle; dans le cadre des travaux pris en compte par le GIEC, de telles projections ont été élaborées à l'aide de réductions d'échelle statistiques ou de modèles régionaux dont les paramètres aux limites sont générés par des modèles climatiques globaux. La redistribution spatiale de chacune de ces contributions individuelles est ensuite calculée en appliquant les effets gravitationnels et rotationnels évoqués précédemment, puis les champs ainsi obtenus sont additionnés pour obtenir la contribution massique totale, en tout point du globe (Figure 2a).

\section{II.2. Contribution stérique}

Avec plus de $90 \%$ du réchauffement global absorbé par les océans, l'expansion thermique aurait contribué pour plus d'un tiers à la hausse globale observée depuis 1971 [Table 13.1 - Church et al., 2013]. Mais à l'instar de la contribution massique, les variations de volume ne sont pas distribuées de manière uniforme. En effet, de nombreux phénomènes affectent le profil vertical de température ou de salinité des océans, faisant varier la densité locale de l'eau de mer de manière cyclique ou durable : évolution des températures de surface, des courants océaniques et des flux thermiques associés, variations de la pression atmosphérique, apports d'eau douce, etc.

En tout point du globe et à tout instant, cette contribution stérique peut être décrite comme la somme d'une composante globale (image de la quantité de chaleur absorbée par les océans) et d'une composante locale (écart par rapport à la moyenne globale occasionné par des transferts d'énergie au sein du système climatique). Dans le cas des plus récents travaux du GIEC, ces deux variables ont été calculées par 21 modèles de circulation générale (Atmosphere-Ocean coupled General Circulation Models, AOGCM et Earth System Models, ESM) répondant au standard CMIP5 [Taylor et al., 2012]. Ceci a permis de générer, en tout point du globe et pour divers scénarios d'émissions dont le scénario RCP8.5 considéré dans cette étude, une valeur moyenne de la contribution stérique (Figure 2b), associée à une fourchette statistique d'incertitude.

\section{II.3. Contribution isostatique}

Les mouvements verticaux du référentiel terrestre associé au niveau marin relatif peuvent être causés par une déformation locale de la croûte terrestre, elle-même engendrée par des variations de charge à sa surface : formation ou fonte de grandes épaisseurs de glace terrestre, augmentation ou diminution conséquente du niveau marin. Fonction de caractéristiques physiques telles que l'épaisseur de la lithosphère et la viscosité du manteau, la relaxation de la croûte terrestre se manifeste par des mouvements de surrection et de subsidence persistant durant plusieurs dizaines de milliers d'années et affectant le niveau marin de plusieurs manières.

En Amérique du Nord et en Europe du Nord, on assiste à un soulèvement de la croûte terrestre à l'aplomb des anciennes calottes glaciaires, et à un affaissement dans leur périphérie. Ainsi à Stockholm, ville située dans la zone recouverte par l'ancienne calotte fenno-scandinave, la contribution isostatique est négative et largement prépondérante par rapport aux autres contributions : les marégraphes y ont enregistré une baisse du niveau marin de $40 \mathrm{~cm}$ au cours du siècle passé. En revanche, dans des villes comme New York ou Vancouver, situées sur le renflement périphérique de l'ancienne calotte laurentide, le taux de subsidence est estimée à près de $15 \mathrm{~cm}$ par siècle, ce qui aggravera la hausse du niveau marin due au changement climatique. En parallèle, la hausse importante et rapide du niveau marin depuis la dernière terminaison glaciaire soumet le plancher océanique à une pression hydrostatique plus élevée et cause son creusement progressif, en particulier le long des marges continentales où le matériel mantellique est repoussé vers la côte et provoque un soulèvement continental [Clark et al., 1978] qui contribue à la diminution du niveau marin relatif.

Ces mécanismes d'ajustement glacio- et hydro-isostatique induisent aussi des variations eustatiques contribuant à accroître le volume des bassins océaniques et donc à faire diminuer le niveau moyen global, à une vitesse d'environ 3 $\mathrm{cm}$ par siècle [Milne et Shennan, 2013]; ce phénomène de siphonage océanique, décrit sur le plan théorique par Mitrovica et Peltier [1991], est directement observable dans le sud du Pacifique, et notamment en Polynésie Française où des récifs coralliens fossiles formés durant l'Holocène tardif surplombent encore aujourd'hui le niveau de la mer [Woodroffe, 2013]. 

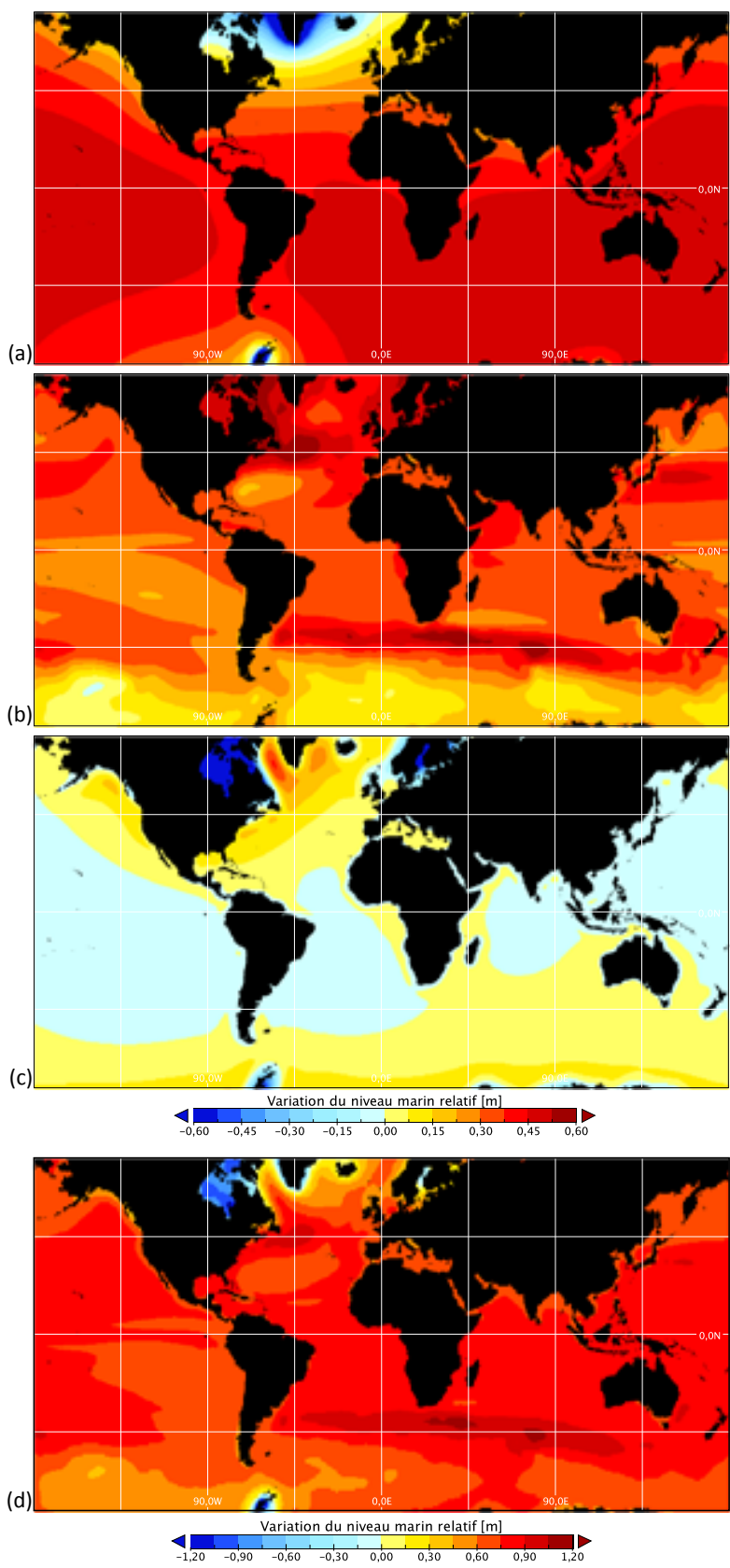

Figure 2 : Variations du niveau marin relatif à l'horizon 2100. Elévation du niveau marin en 2100 par rapport à la période de référence 1986-2005, pour le scénario RCP8.5. (a) Contribution massique. (b) Contribution stérique. (c) Contribution isostatique. (d) Variation totale. Figures originales élaborées à partir des données numériques compilées par le GIEC [Supplementary Material - Church et al., 2013].

Ces ajustements pouvant être considérés comme linéaires à l'échelle centennale, les taux de variations actuels sont extrapolés pour calculer la contribution isostatique à l'horizon 2100. Pour les déterminer, le GIEC a effectué la moyenne des résultats obtenus grâce à deux modèles de glaces terrestres, un modèle développé à l'Australian National University [Lambeck et al., 1998] et le modèle ICE-5G [Peltier, 2004], chacun associés à un modèle viscoélastique $1 \mathrm{D}$ décrivant la rhéologie de la Terre. (Figure 2c).

\section{COMPARAISON DES PROJECTIONS RÉGIONALES}

\section{III.1. Poids relatif des différentes contributions}

Sur la base des données compilées par le GIEC dans son dernier rapport, les valeurs moyennes de chacune des trois contributions en 2100 par rapport à la période de référence 1986-2005 ont été calculées et comparées entre elles pour dix sites côtiers d'outremer et de métropole (Figure 3).

En considérant le poids relatif des différentes contributions, les sites peuvent être divisés en deux groupes : la zone intertropicale (Nouméa, Mata-Utu, Papeete, Fort-de-France, Cayenne, Mamoudzou, Saint-Denis) et les latitudes moyennes (Port-aux-Français au sud, Saint-Pierre et Brest au nord).

En zone intertropicale, la contribution massique devrait être importante ( $40 \mathrm{~cm}$ en moyenne) et très supérieure à la moyenne globale pour tous les sites. En effet, aux basses latitudes, les contributions respectives de la calotte du Groenland et de l'Antarctique se cumulent et sont toutes les deux proches de leur maximum global. Dans cette même zone, la contribution stérique devrait aussi être importante (30 $\mathrm{cm}$ en moyenne) mais proche de la moyenne globale. Sur ce point, on notera aussi que dans le Pacifique, et en particulier à Mata-Utu, le niveau marin affiche une forte variabilité à l'échelle décennale [Alory et Delacroix, 1999] ; de nature stérique, celle-ci est principalement causée par l'oscillation australe et le phénomène El Niño. Bien que ces variations soient masquées dans les projections de hausse à l'horizon 2100 - qui sont moyennées sur 20 ans en début et fin de période - il serait judicieux de considérer leur amplitude dès lors que le risque de submersion est évalué selon une approche probabiliste, en combinaison avec d'autres facteurs de surcote. Enfin, les effets des ajustements isostatiques en zone intertropicale seront négligeables et légèrement négatifs compte tenu de l'effet de siphonage océanique mentionné précédemment. La seule exception concerne le site de Fort-de-France, pour lequel la contribution isostatique sera légèrement positive ; la zone des Caraïbes, située en périphérie de l'ancienne calotte laurentide, connaît en effet un épisode de subsidence.

Aux latitudes moyennes de l'hémisphère nord, la contribution massique s'avère plus faible qu'à l'équateur, les effets gravitationnels étant plus sensibles à proximité des grandes masses de glace - en l'occurrence, la calotte du Groenland et les glaciers de la zone Arctique. Ainsi à Saint-Pierre, la contribution individuelle de la calotte du Groenland est légèrement négative, et presque nulle à Brest. Malgré une influence plus fortement ressentie dans l'hémisphère nord, et sauf instabilité majeure, la contribution de la calotte de l'Antarctique devrait quant à elle demeurer assez faible au cours du siècle à venir. Par contraste, la contribution stérique dans 1'Atlantique Nord et le long des parallèles $45^{\circ} \mathrm{N}$ and $45^{\circ} \mathrm{S}$ sera supérieure à la valeur moyenne globale, traduisant une évolution sensible des flux de chaleur et de salinité entre les hautes et basses latitudes d'ici la fin du siècle. Cette contribution devrait être substantielle à Brest et à Port-aux-Français, et même prépondérante à Saint-Pierre-et-Miquelon, archipel baigné par le courant du Labrador. Enfin, ces trois sites se trouvent en périphérie de régions ayant connu d'importants épisodes de déglaciation et subissent donc un ajustement isostatique positif, quoique faible. 


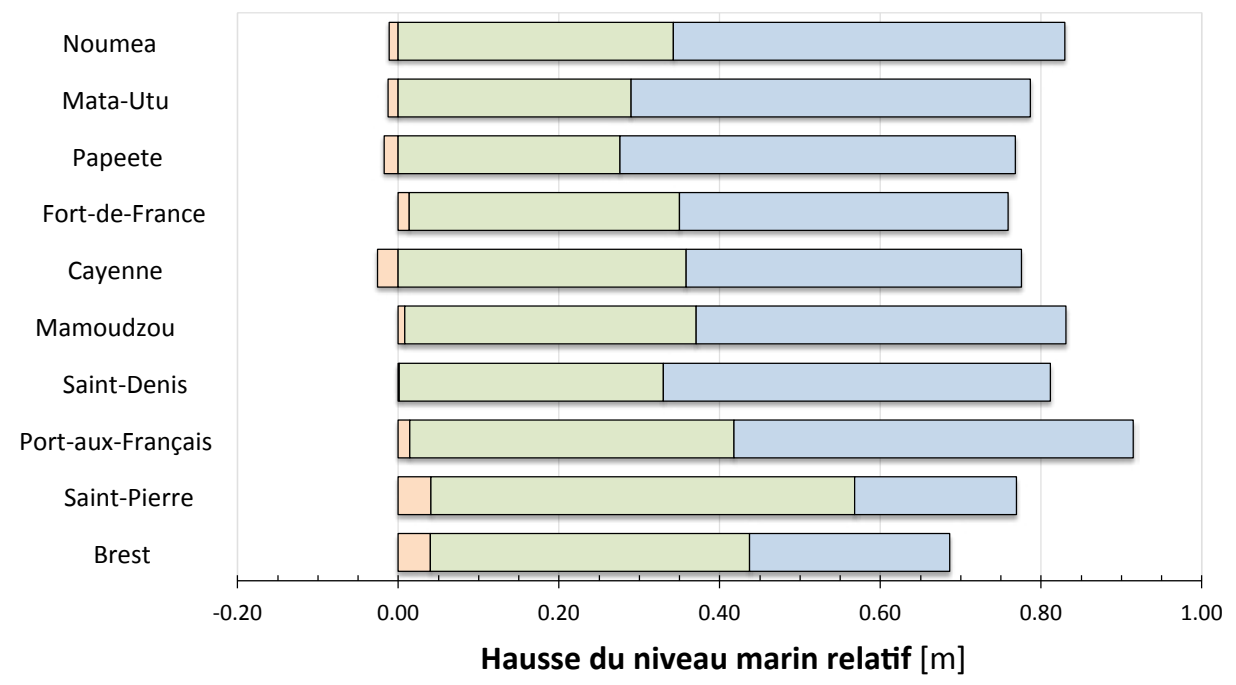

Figure 3 : Contributions à la variation du niveau marin sur les côtes françaises à l'horizon 2100. Les valeurs moyennes des contributions (en m) en 2100 par rapport à la période de référence 1986-2005 sont comparées pour dix sites d'outremer et de métropole, dans le scénario RCP8.5 ; contribution stérique (en vert), contribution massique (en bleu) et contribution isostatique (en orange).

\section{III.2. Sensibilité des projections aux erreurs et incertitudes}

$\mathrm{Au}$ delà des incertitudes relatives à la modélisation des émissions anthropiques ou des composantes du système climatique, de nombreuses sources d'erreurs et d'incertitudes sont associées aux codes de calculs et modèles utilisés pour établir des projections globales ou régionales du niveau marin.

En premier lieu, la prise en compte de la dynamique des glaces dans les récentes évaluations du GIEC constitue une avancée majeure mais suscite aussi de nombreuses interrogations. L'une des plus importantes sources d'incertitude concerne le comportement des glaciers et plateformes de glace en périphérie du continent Antarctique, notamment dans le secteur ouest et à proximité de la péninsule. Leur instabilité pourrait entraîner une hausse globale du niveau marin supérieure aux projections [Paragraphe E.6 - IPCC, 2013] ; selon le GIEC, cette contribution additionnelle «ne dépasserait pas quelques dixièmes de mètre d'élévation du niveau des mers au cours du XXIe siècle». En dépit de la formulation employée, la perspective d'une telle hausse n'est pas anodine, notamment pour le dimensionnement des mesures d'adaptation, et elle introduit une source d'incertitude majeure dans les projections. En outre, un calcul de sensibilité effectué dans le cadre de cette étude montre que les côtes françaises seraient plus exposées que la moyenne aux conséquences d'un tel phénomène, mais de manière relativement uniforme. A titre d'illustration, la fonte d'un volume équivalent à $10 \mathrm{~cm}$ de niveau moyen global se traduirait par une hausse d'environ $15 \mathrm{~cm}$ pour l'ensemble des sites examinés, excepté à Port-aux-Français où le niveau de la mer ne varierait pas.

En revanche, une fonte supérieure aux estimations de la calotte du Groenland conduirait à un accroissement considérable des écarts de projections entre la métropole et les territoires ultramarins. Ainsi, dans les projections les plus pessimistes du GIEC, la contribution individuelle du Groenland à la hausse du niveau moyen global pourrait être de $28 \mathrm{~cm}$ d'ici 2100 ; ceci se traduirait par une contribution de seulement $8 \mathrm{~cm}$ à Brest, et de 30 à $35 \mathrm{~cm}$ dans le reste des DOM-COM, Saint-Pierre-et-Miquelon excepté. Or de récentes observations suggèrent que la contribution future de la calotte du Groenland à la hausse du niveau marin serait sous-estimée du fait de la méconnaissance de certains mécanismes tels que la lubrification de la base de la calotte par la décharge des lacs supraglaciaux, les rétroactions positives associées à la relation altitude-température ou encore l'action thermique de l'océan à la base des glaciers, dans une zone périphérique encore mal topographiée. [Leeson et al., 2014 ; Csatho et al., 2014 ; Morlighem et al., 2014].

Concernant la contribution stérique, la généralisation du recours aux ESM et l'amélioration de la résolution spatiale des modèles devrait permettre d'affiner les projections, mais certains phénomènes physiques ne sont pas encore correctement modélisés à ce stade. Par exemple, les modèles climatiques globaux et les modèles régionaux décrivant le comportement des calottes glaciaires ne sont pas couplés [Paragraphe 13.1.4.1 - Church et al., 2013). De ce fait, l'incidence de la fonte des glaces terrestres est prise en compte de manière incomplète alors qu'elle affecte localement la contribution stérique. En outre, cet apport d'eau douce pourrait perturber la circulation thermohaline et modifier les transferts de chaleur entre hautes et basses latitudes [Paragraphe E.4 - IPCC, 2013] ; ceci influerait nécessairement sur les écarts de projections entre la métropole et les territoires d'outremer, dans des proportions qui restent difficiles à évaluer dans l'état actuel des connaissances.

Enfin, modéliser les processus d'isostasie avec une résolution spatiale suffisante requiert de formuler des hypothèses précises non seulement sur l'historique des changements ayant affecté les glaces terrestres, mais aussi sur la structure interne de la Terre. Or, à la différence des autres contributions, seuls deux modèles ont été considérés par le GIEC pour déterminer la contribution isostatique, et ceux-ci font apparaître des écarts parfois notables dans les régions qui subissent un fort rebond post-glaciaire ; c'est notamment le cas à Brest et Saint-Pierre-et-Miquelon, sites pour lesquels les projections s'avèrent particulièrement sensibles au paramétrage des modèles. A titre d'exemple, les résultats des deux modèles d'ajustement isostatique retenus par le GIEC affichent une différence de $10 \mathrm{~cm}$ par siècle à Saint-Pierre. 


\section{III.3. Comparaison de l'aléa 2100 et des projections régionales}

Actuellement fixée à $60 \mathrm{~cm}$ pour l'ensemble des côtes françaises, la valeur de l'aléa 2100 a été déterminée en appliquant le critère suivant aux conclusions du quatrième rapport du GIEC : fourchette haute des projections (valeur haute de l'intervalle de confiance à 5-95\%) dans le scénario d'émissions de GES le plus défavorable (scénario SRES A1FI). En Figure 4 sont présentées des projections régionales calculées en appliquant un critère équivalent aux données compilées par le GIEC dans son cinquième rapport (valeur haute de l'intervalle de confiance à 5-95\%, scénario RCP 8.5, cf. Figure 1).

En première analyse, il ressort que la hausse du niveau marin le long des côtes françaises présente une variabilité modérée mais non négligeable; si l'on excepte Port-aux-Français, la dispersion des projections serait proche de $20 \mathrm{~cm}$ pour l'ensemble des sites examinés. Notons aussi que la métropole sera moins affectée que l'ensemble des territoires d'outremer ; cette observation reste valable dans les autres scénarios d'émission de GES, quelque soit l'amplitude de la hausse globale. Enfin, il convient de souligner que dans le scénario pessimiste envisagé ici, les projections régionales excéderaient l'aléa 2100 d'au moins $80 \%$ pour l'ensemble des sites d'outremer.

\section{CONCLUSION}

Se basant sur des projections pessimistes établies en 2007, la réglementation relative au risque de submersion marine envisage une hausse maximale de $60 \mathrm{~cm}$ sur l'ensemble des côtes françaises d'ici la fin du siècle. Mais le dernier rapport du GIEC prévoit une élévation globale du niveau des mers de $98 \mathrm{~cm}$ dans un scénario similaire, ce qui implique que le risque de submersion à l'horizon 2100 est désormais sous-estimé.

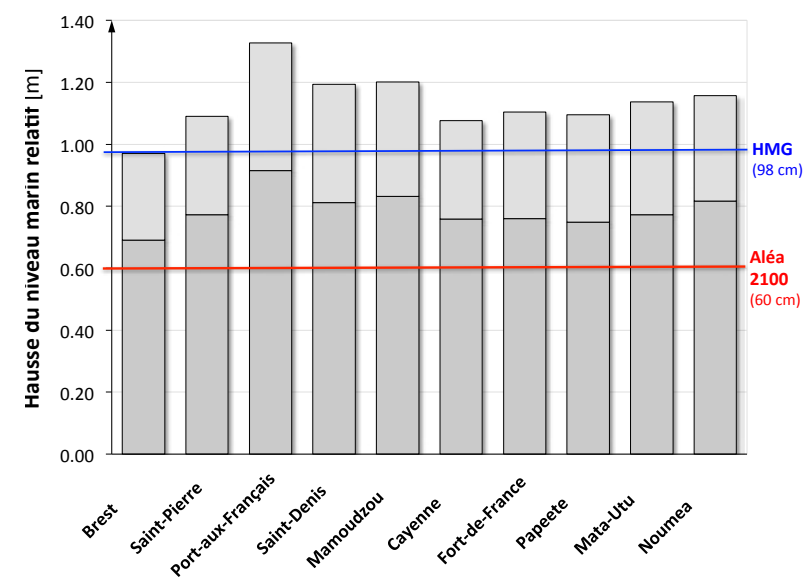

Figure 4 : Projections de la hausse du niveau marin sur les côtes françaises à l'horizon 2100. Valeurs moyennes (barres en gris foncé, en $m$ ) et valeurs hautes de l'intervalle de confiance à 5-95\% (barres en gris clair, en m) de l'élévation du niveau marin en 2100 par rapport à la période de référence 1986-2005 pour dix sites d'outremer et de métropole, dans le scénario RCP8.5 ; ces projections sont comparées à la valeur haute de la hausse du niveau global moyen sur la même période (HMG, en bleu) et à l'aléa 2100 (en rouge).
De surcroît, les variations locales du niveau marin peuvent s'écarter significativement de la hausse moyenne globale, en réponse à des processus physiques affectant la redistribution spatiale des masses et des densités. L'analyse des projections régionales montre que le littoral français sera affecté de manière défavorable par cette variabilité : dans le scénario étudié, une hausse sensiblement égale à la moyenne globale est attendue sur la façade Atlantique de la métropole, mais cette moyenne serait dépassée partout ailleurs dans les territoires d'outremer ; elle pourrait notamment atteindre $120 \mathrm{~cm}$ dans des îles comme Mayotte ou La Réunion.

La réglementation en matière de prévention des risques littoraux prévoyant explicitement d'intégrer l'évolution des connaissances scientifiques sur le climat, il conviendrait d'actualiser l'expertise nationale et de réviser le corpus réglementaire à la lumière des plus récents travaux du GIEC. En première approche, il est suggéré d'augmenter la valeur de l'aléa 2100 à $100 \mathrm{~cm}$, voire de formuler des hypothèses régionalisées de manière à prendre en compte l'exposition plus importante des territoires ultramarins.

Il convient enfin de rappeler que le risque de submersion marine résulte de plusieurs facteurs, et qu'en conjonction avec l'évolution de la fréquence et de l'intensité des évènements climatiques extrêmes, la hausse progressive du niveau marin contribuera nécessairement à une augmentation de ce risque. Ainsi Kopp et al. [2014] ont montré que dans l'hypothèse d'une hausse répondant au scénario RCP8.5, la probabilité d'occurrence d'un événement centennal de submersion marine, aux conséquences potentiellement désastreuses, serait multipliée par 9 dans une ville comme New York. Une étude similaire s'appuyant sur des méthodologies et données existantes [Kergadallan 2013 ; Lecacheux et al., 2013] permettraient d'estimer l'évolution des périodes de retour dans les DOM-COM à l'horizon 2100 et de réévaluer le risque en conséquence.

\section{REMERCIEMENTS}

L'auteur remercie Glenn Milne pour ses précieux commentaires concernant les travaux du GIEC, l'Integrated Climate Data Center de l'université de Hamburg qui a diffusé au format netCDF les données numériques relatives au niveau de la mer compilées par le GIEC, ainsi que le groupe de recherche en dynamique du système Terre de l'université d'Ottawa qui a mis à disposition les ressources informatiques nécessaires pour exploiter ces données.

\section{RÉFÉRENCES}

Alory G. et T. Delacroix (1999) - Climatic variability in the vicinity of Wallis, Futuna, and Samoa islands (13 degrees-15 degrees S, 180 degrees-170 degrees W). Oceanologica Acta. 22(3), 249-263.

Bellard C., C. Leclerc et F. Courchamp (2013) - Potential impact of sea level rise on French islands worldwide. Nature Conservation. 5, 75-86.

Church J.A., P.U. Clark A. Cazenave J.M. Gregory S. Jevrejeva A. Levermann M.A. Merrifield G.A. Milne R.S. Nerem P.D. Nunn A.J. Payne W.T. Pfeffer D. Stammer et A.S. Unnikrishnan (2013) - Sea Level Change. Climate Change 2013: The Physical Science Basis. Contribution of Working Group I to the Fifth Assessment Report of the Intergovernmental Panel on Climate Change [Stocker, T.F., D. Qin, G.-K. Plattner, M. Tignor, S.K. Allen, J. Boschung, A. Nauels, Y. Xia, V. Bex and P.M. Midgley (eds.)]. Cambridge University Press, Cambridge, United Kingdom and New York, NY, USA. 
Clark J.A., W.E. Farrell et W.R. Peltier (1978) - Global changes in postglacial sea level: A numerical calculation. Quaternary Research. 9(3), 265-287.

Csatho B., A. Schenk C. Van Der Veen G. Babonis K. Duncan S. Rezvanbehbahani M. Van Den Broeke S. Simonsene S. Nagarajan, et J. VAn Angelen (2014) — Laser altimetry reveals complex pattern of Greenland Ice Sheet dynamics. Proceedings of the National Academy of Sciences. 111(52), 18478-18483.

Duvat V., A. Magnan G. Mossot M. Galliot S. Mondon et B. REYSSET (2012) - Les outremer face au défi du changement climatique. Rapport au Premier Ministre et au Parlement, sous la direction de l'Observatoire National sur les Effets du Réchauffement Climatique. La Documentation Française. 2012.

Feuillet T., E. Chauveau et L. Pourinet (2012) - Xynthia est-elle exceptionnelle? Réflexions sur l'évolution et les temps de retour des tempêtes, des marées de tempête, et des risques de surcotes associés sur la façade atlantique française. Norois. 1/2012 (222), 27-44.

Kergadallan X. (2013) - Analyse statistique des niveaux d'eau extrêmes, environnements maritime et estuarien. Centre d'études techniques maritimes et fluviales.

Kopp R.E., R.M. Horton C.M. Little J.X. Mitrovica M. Oppenheimer D.J. Rasmussen B.H. Strauss, et C. Tebaldi (2014) - Probabilistic 21st and 22nd century sea-level projections at a global network of tide-gauge sites. Earth's Future. 2, 383-406.

Lambeck K., C. Smither, et P. Johnston (1998) - Sea-level change, glacial rebound and mantle viscosity for northern Europe. Geophysical Journal International. 134, 102-144.

Leeson A. A., A. Shepherd K. Briggs I. Howat X. Fettweis M. Morlighem Et E. Rignot (2014) - Supraglacial lakes on the Greenland ice sheet advance inland under warming climate. Nature Climate Change. 5, 51-55.

IPCC (2013) - Summary for Policymakers. Climate Change 2013: The Physical Science Basis. Contribution of Working Group I to the Fifth Assessment Report of the Intergovernmental Panel on Climate Change [Stocker, T.F., D. Qin, G.-K. Plattner, M. Tignor, S.K. Allen, J.Boschung, A. Nauels, Y. Xia, V. Bex and P.M. Midgley (eds.)]. Cambridge University Press, Cambridge, United Kingdom and New York, NY, USA.1-30.

Lecacheux S., T. Bulteau R. Pedreros E. Delvallee et F. Paris (2013) — Projet ARAI3 : évaluation probabiliste des houles et des surcotes cycloniques en Polynésie Française. Rapport du Bureau de Recherches Géologiques et Minières BRGM/ $R P-61888-F R$.
Meehl G.A., T.F. Stocker W.D. Collins P. Friedlingstein A.T. Gaye J.M. Gregory A. Kitoh R. Knutti J.M. Murphy A. Noda S.C.B. Raper I.G. Watterson A.J. Weaver et Z.-C. Zhaо (2007) - Global Climate Projections. Climate Change 2007: The Physical Science Basis. Contribution of Working Group I to the Fourth Assessment Report of the Intergovernmental Panel on Climate Change [Solomon, S., D. Qin, M. Manning, Z. Chen, M. Marquis, K.B. Averyt, M. Tignor and H.L. Miller (eds.)]. Cambridge University Press, Cambridge, United Kingdom and New York, NY, USA.

Milne G.A. ET I. SHENNAN (2013) — Isostasy : Glaciation-Induced Sea-Level Change. Encyclopedia of Quaternary science. 452-459.

Milne G.A. ET J.X. Mitrovica (1998) - Postglacial sea-level change on a rotating Earth. Geophysical Journal International. 133, 1-19.

Ministere de L'Ecologie, du Developpement Durable, des TRANSPORTS ET DU LOGEMENT (2011) - Circulaire relative à la prise en compte du risque de submersion marine dans les plans de prévention des risques naturels littoraux, 27 juillet 2011

Mitrovica J.X. et W.R. Peltier (1991) - On postglacial geoid subsidence over the equatorial oceans. Journal of Geophysical Research. 96, 20053-20071.

Morlighem M., E. Rignot J. Mouginot H. Seroussi et E. Larour (2014) - Deeply incised submarine glacial valleys beneath the Greenland Ice Sheet. Nature Geoscience. 7(6), 418-422.

Observatoire National Sur Les Effets Du Rechauffement Climatique (2010) - Prise en compte de l'élévation du niveau de la mer en vue de l'estimation des impacts du changement climatique et des mesures d'adaptation possibles. Synthèse publiée sous la direction de P.F. Chevet.

Peltier W.R. (2004) - Global glacial isostasy and the surface of the ice-age earth: The ICE-5G (VM2) model and GRACE. Annual Review of Earth and Planetary Sciences. 32, 111-149.

Tamisiea M.E., J.X. Mitrovica J.L. Davis G.A. Milne (2003) Solid Earth Physics: Long Wavelength Sea Level and Solid Surface Perturbations Driven by Polar Ice Mass Variations: Fingerprinting Greenland and Antarctic Ice Sheet Flux. Space Science Reviews. 108, 81-93.

Taylor K.E., R.J. Stouffer et G.A. Meehl (2012) - An Overview of CMIP5 and the Experiment Design. Bulletin of the American Meteorological Society. 93, 485-498.

Woodroffe C.D. (2013) - Coral Records of Relative Sea-Level Changes. In. Encyclopedia of Quaternary Science. 409-418. 